\title{
Two-site simultaneous observations of solar-like stellar oscillations in radial velocities with a Fabry-Pérot calibration system ${ }^{\star}$
}

\author{
J.-L. Bertaux ${ }^{1}$, J. Schmitt ${ }^{1,3}$, J.-C. Lebrun ${ }^{1}$, F. Bouchy ${ }^{2}$, and S. Guibert ${ }^{1}$ \\ 1 Service d'Aéronomie du CNRS, BP 3, 91371 Verrières-le-Buisson, France \\ 2 Observatoire de Genève, $51 \mathrm{Ch}$. des Maillettes, 1290 Sauverny, Switzerland \\ 3 Observatoire de Haute Provence, 04870 Saint Michel L'Observatoire, France
}

Received 15 July 2002 / Accepted 1 April 2003

\begin{abstract}
We report simultaneous measurements of the radial velocity $V_{\mathrm{r}}$ of a star, $\zeta$ Herculis, with two different telescopes and spectrometer systems, both located at Observatoire de Haute Provence, France. We believe that we detected for the first time in a solar type star a correlated signal in the time series of $V_{\mathrm{r}}$ observed simultaneously with the two different instruments, due to the stellar oscillations. The objective of our observations was to compare the performance of the two systems, for the detection of stellar oscillations (astero-seismology). Both systems use a large portion of the visible spectrum of the star to increase the number of spectral lines on which to compute variations of $V_{\mathrm{r}}$, and a white-light channelled spectrum produced by a Fabry-Pérot as a calibration of spectrometer drifts. The two calibration systems that we designed and implemented are however different. With the $193 \mathrm{~cm}$ telescope, we calibrated the ELODIE spectrometer with a fixed Fabry-Pérot, while for the EMILIE spectrometer, we use the full AAA (Astronomical Absolute Accelerometer) system of Pierre Connes, with a tunable Fabry-Pérot and various servo-controls. We first show, with three stars observed with ELODIE (Procyon, $\eta$ Cas and $\zeta$ Her), that the auto-correlation function on a single-night time series is clearly significant when the periodogram shows significant peaks (Procyon and $\zeta$ Her), while for $\eta$ Cas, nothing significant is seen in the periodogram nor in the auto-correlation function. Then we show evidence of the correlation of the two simultaneous, but independent, time-series obtained on a single night on $\zeta$ Her, that we believe is the first reported clear case of simultaneous detection of stellar oscillations (solar type), which paves the way to future multi-site observations with a network of longitude spread telescopes. Finally, the observed fluctuations are compared, yielding an estimate of the instrumental (plus atmospheric effects) short term random error: $<2.1 \mathrm{~m} \mathrm{~s}^{-1}$ for EMILIE + AAA system, and $<2.9 \mathrm{~m} \mathrm{~s}^{-1}$ for ELODIE + fixed Fabry-Pérot system.
\end{abstract}

Key words. stars: oscillations - stars: individual: $\zeta$ Her A - techniques: spectroscopic

\section{Introduction}

Doppler astero-seismology refers to an observational method to probe the interior of stars in which a time-series of stellar radial velocities measurements is analyzed to find periods and amplitudes of disc integrated stellar oscillations, in the range around $1 \mathrm{mHz}$ and $0.1-1 \mathrm{~m} \mathrm{~s}^{-1}$ respectively. On the other hand, photometric methods requiring a few $10^{-6}$ relative accuracy maintained over several days have been pushed to their limit with CCD differential photometry. Using a global network of several 4-m telescopes, Gilliland et al. (1993) observed during $156 \mathrm{hrs}$ the stars in the cluster M 67, without positive results for solar-like oscillations.

Send offprint requests to: J.-L. Bertaux,

e-mail: jean-loup.bertaux@aerov.jussieu.fr

* Based on observations obtained at the Observatoire de Haute-Provence (CNRS, France).
Atmospheric scintillations spoil photometric measurements, and only space-based instrumentation will return the full potential of the photometric method. The first space-borne attempt with EVRIS instrument (Baglin 1994) failed when the Mars 96 probe was destroyed at launch; several space missions will be dedicated to this method in the near future (e.g., French COROT, Canadian MOST, Danish MONS missions).

Many early spectroscopic attempts failed also to produce convincing results using Balmer-line equivalent widths (Kjeldsen et al. 1999), a resonance-cell spectrometer (Gelly et al. 1986), a magneto-optical filter (Innis et al. 1991; Bedford et al. 1995), a Fabry-Pérot interferometer (Ando et al. 1988).

For slow rotator stars (small $v \sin i$ ), recording a large portion of the visible spectrum at high spectral resolution allows one to achieve a better accuracy on the derivation of the Doppler shift, thanks to the many Fraunhofer absorption lines recorded simultaneously. The most important need is a 
good short term stability of the high resolution spectrometer, together with an excellent wavelength calibration system.

The first results for the detection of solar-like oscillations were obtained in November 1998 for Procyon by Martic et al. (1999), using the fiber-fed cross-dispersed ELODIE spectrometer at Observatoire de Haute Provence (OHP), in which a channeled spectrum provided by a Fabry-Pérot served as a calibration system, and will be described below in further details. The iodine absorption cell technique was later successfully used in the Southern hemisphere (with the $3.9 \mathrm{~m}$ Anglo Australian Telescope), yielding some evidence for solar-like oscillations in star $\beta$ Hyi (Bedding et al. 2001), and most recently the thorium-argon calibration system of the CORALIE spectrometer on the Euler swiss telescope at La Silla (Chili) brought excellent results on $\alpha$ Centauri (Bouchy et al. 2001) and confirmed the results on $\beta$ Hyi (Carrier et al. 2001) found at the AAT (Australia) in a two-site coordinated campaign in June 2000, after the observations reported here. It is striking to see that all three systems which provided the best and most recent results in asteroseismology were in fact originally constructed for high precision radial velocity measurements, not for asteroseismology, but rather oriented toward the search of extra-solar planets. The fourth system, the Absolute Astronomical Accelerometer (AAA), invented by Pierre Connes, is no exception, though Connes (1985) mentioned explicitly asteroseismology as a branch of astrophysics which would also benefit from high precision radial velocities of stars.

In fact, while these two domains of astronomy are connected from a methodological point of view, and seem disconnected scientifically, it is quite possible that the study of extra-solar planetary systems would benefit from asteroseismology. Indeed, on a known system, it is important to characterize the host star as well as possible and in particular its age. In some range of ages where other methods are inaccurate (stellar evolution models), the determination of the large separation $\Delta v$ and the small separation $\delta v$ of oscillations modes may provide a unique way to determine the thickness of the convection zone and the age of the star (Christensen-Dalsgaard 1998). Therefore, we expect in the future that stars hosting planets will be a prime target of interest for asteroseismology.

In this paper, we report what we believe to be the first simultaneous detection of stellar oscillations with two different telescopes and instruments, paving the way for multi-sites observations of the same star from various locations spread in longitude, to alleviate the problem caused by day/night interruption experienced in a single site location. Though the two telescopes, both located at Observatoire de Haute Provence (France), are only distant by $400 \mathrm{~m}$, most of the difficulties and technicalities in analyzing multi-site observations are present when examining these two data sets.

The first equipment is the ELODIE cross-dispersed spectrometer, fiber fed from the Cassegrain focus of the $1.93 \mathrm{~m}$ telescope. A series of 15 nights were dedicated to the search for stellar oscillations of star $\zeta$ Herculis $\left(m_{\mathrm{v}}=2.807\right)$ (Martic et al. 2001). The wavelength calibration system was a specially implemented Fabry-Pérot interferometer.
The second equipment is the EMILIE cross-dispersed spectrometer, fiber fed from the Coudé focus of the $1.52 \mathrm{~m}$ telescope of OHP, with the full AAA calibration system. During the night from 14 to 15 May 2000, the star $\zeta$ Her was also observed with EMILIE.

In the next section, we describe briefly each of the two systems, and the data analysis done to obtain the times-series of radial velocity measurements $\left(V_{\mathrm{r}}\right)$. In the third section, we analyze three time series of $V_{\mathrm{r}}$ obtained with ELODIE with the autocorrelation technique, and conclude that, when some power is present in the periodogram analysis of the time series, the oscillations can be seen "with the naked eye" in the time series. In Sect. 4, the results on $\zeta$ Her obtained during one night with EMILIE are presented. In Sect. 5, we obtain a correlation of the two independent time series, and these results are discussed in the last section.

\section{Description of spectrometers and calibrations systems}

\subsection{The ELODIE spectrometer}

This spectrometer was designed by Baranne (Baranne et al. 1966) and constructed at OHP. Its design was optimized to measure stellar radial velocities $\left(V_{\mathrm{r}}\right)$ with a high accuracy, in order to improve on the previous CORAVEL instrument, for the programme initiated by Michel Mayor (Observatoire de Genève). In this cross-dispersed echelle spectrograph, the stellar light is fed from the $1.93 \mathrm{~m}$ focus through a $100 \mu \mathrm{m}$ optical fiber, and the spectrum spread over 67 echelle orders, each covering $5.25 \mathrm{~nm}$, covering a total wavelength domain from 390.6 to $681.1 \mathrm{~nm}$, at a resolution of $\sim 42000$. A second optical fiber whose 67 orders are interleaved with the stellar spectrum can be used to record simultaneously the sky background, or to record the calibration spectrum provided by a thorium-argon discharge lamp, on the same CCD (Tk 1024× 1024, $24 \mu \mathrm{m}$ pixels). A standard reduction package extracts the stellar spectrum from the CCD frame and provides the absolute radial velocity of the star, by correlation with standard masks of known spectral features.

This ELODIE spectrograph was also originally designed to accommodate, with a second entry port, the AAA calibration system (described below). However, when ELODIE began systematic observations in 1995, the AAA system developed at Service d'Aeronomie was not ready yet. And when it was later completed, it was felt that any mechanical intervention in the ELODIE spectrometer could produce detrimental interference with the on-going long term programme of $V_{\mathrm{r}}$ variations monitoring of Observatoire de Genève, which led to the discovery of the first extra-solar planet around another star (Mayor \& Queloz 1995).

Nevertheless, we proposed (Bertaux 1994, Action Incitative INSU) to use the ELODIE spectrograph for asteroseismology, with an alternate calibration system inspired by, but simpler than, the AAA concept, and not requiring any intervention inside the spectrograph. The thorium-argon source was replaced by a closely-spaced channeled spectrum provided by white light from a tungsten filament through a 


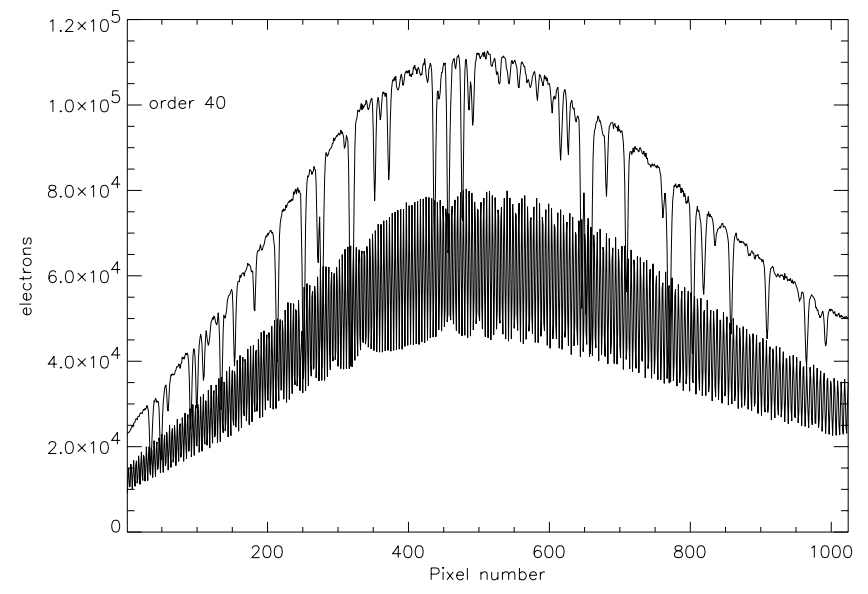

Fig. 1. Example of stellar and Fabry-Perot spectrum from a single order in the ELODIE spectrometer. (from Martic et al. 1999).

fixed Fabry-Pérot in ZERODUR, with a low finesse (Fig. 1), implemented by Schmitt in 1995. The Fabry-Pérot etalon has a thickness of $6 \mathrm{~mm}$ and produces a comb of fringes with a spacing of about 3 pixels. It is temperature controlled to a precision of $0.01 \mathrm{~K}$. The advantage of the channeled spectrum over the thorium-argon spectrum is to provide many more spectral features, uniformly spread over the whole CCD which allows one to better track all drifts of the spectrometer and correct for them. This is however at the expense of an absolute determination of $V_{\mathrm{r}}$, which is not necessary for asteroseismology.

Our first observations on a bright star ( $\psi$ Ursa Majoris (HR 4335, $m_{\mathrm{v}}=3.0, \mathrm{~K}$ III) were conducted in 1995. The $V_{\mathrm{r}}$ measurements (Connes et al. 1996) showed some fluctuations that seemed of instrumental origin. Consequently, the temperature control of the ELODIE room was improved (the on-off regulation system within $\pm 0.5{ }^{\circ} \mathrm{C}$ was replaced by a proportionnal regulation system). In 1998, another improvement was obtained by introducing a double-scrambler on the secondary port initially dedicated to the AAA system, and now used in a routine fashion to mitigate the effect of variable seeing.

All this led finally in November 1998 to the first detection of stellar oscillations in Procyon, and no detection in $\eta$ Cas (Martic et al. 1999). However, a careful re-analysis of our observations prior to 1998 indicate that the recorded fluctuations, first attributed to an instrumental effect, included also some genuine stellar oscillations (Martic et al. 2001). Some of the 1998 results will be discussed in Sect. 3 .

\subsection{The EMILIE spectrometer and the AAA system}

When it became clear that it would be difficult to implement the full AAA system on the ELODIE spectrometer without interfering with the programme of Observatoire de Geneve, Connes decided to build EMILIE, a new cross-dispersed spectrometer, dedicated to accommodate the full AAA system. EMILIE is described in details in Bouchy (1999), and the actual AAA system and performances in Schmitt \& Bertaux (1997, 2003).
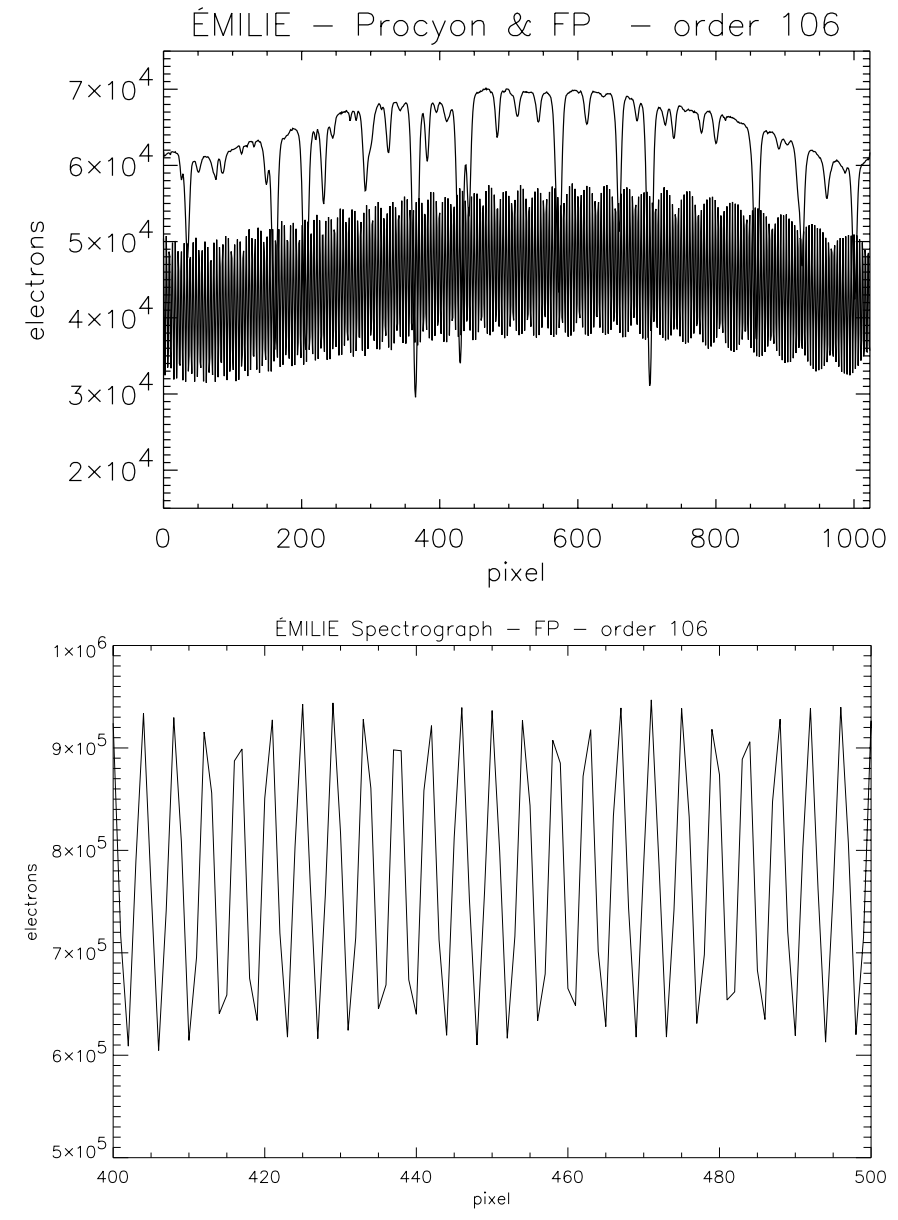

Fig. 2. Upper panel: example of stellar and Fabry-Pérot spectra for the EMILIE spectrometer. Lower panel: zoom on the channeled FabryPérot spectrum showing 4 pixels per fringe.

After construction and preliminary testings at Service d'Aéronomie, EMILIE and the AAA system was moved to OHP in March 1999, installed in the coudé room of the telescope, and coupled to the coudé focus of the $1.52 \mathrm{~m}$ telescope.

The main differences between EMILIE/AAA system and the ELODIE/FP system are:

- there is only one optical fiber, through which the star and the calibration spectrum are fed in alternance. This requires an even higher stability of the spectrometer, achieved by an even better thermal control of the whole spectrometer;

- an image slicer is used to increase the spectral resolution to $\sim 150000$; the $V_{\mathrm{r}}$ size of a spectel is $1.3 \mathrm{~km} \mathrm{~s}^{-1}$ versus $3 \mathrm{~km} \mathrm{~s}^{-1}$ for ELODIE;

- the CCD camera used a front side illuminated CCD and camera controller C-100 from Photometrics;

- the image of the star is stabilized on the entrance of the fiber $\left(100 \mu \mathrm{m}\right.$, accepting $2.7^{\prime \prime}$ on the sky) with a specially designed FLAG system (Fiber Locked Auto-Guidance, Bouchy \& Connes 1999). The fiber is used as a scrambler;

- the calibration spectrum is also a channeled spectrum produced by white light through a Fabry-Pérot (cf. Fig. 2), but the thickness of this FP is tunable, and controlled by a system of laser diodes. 


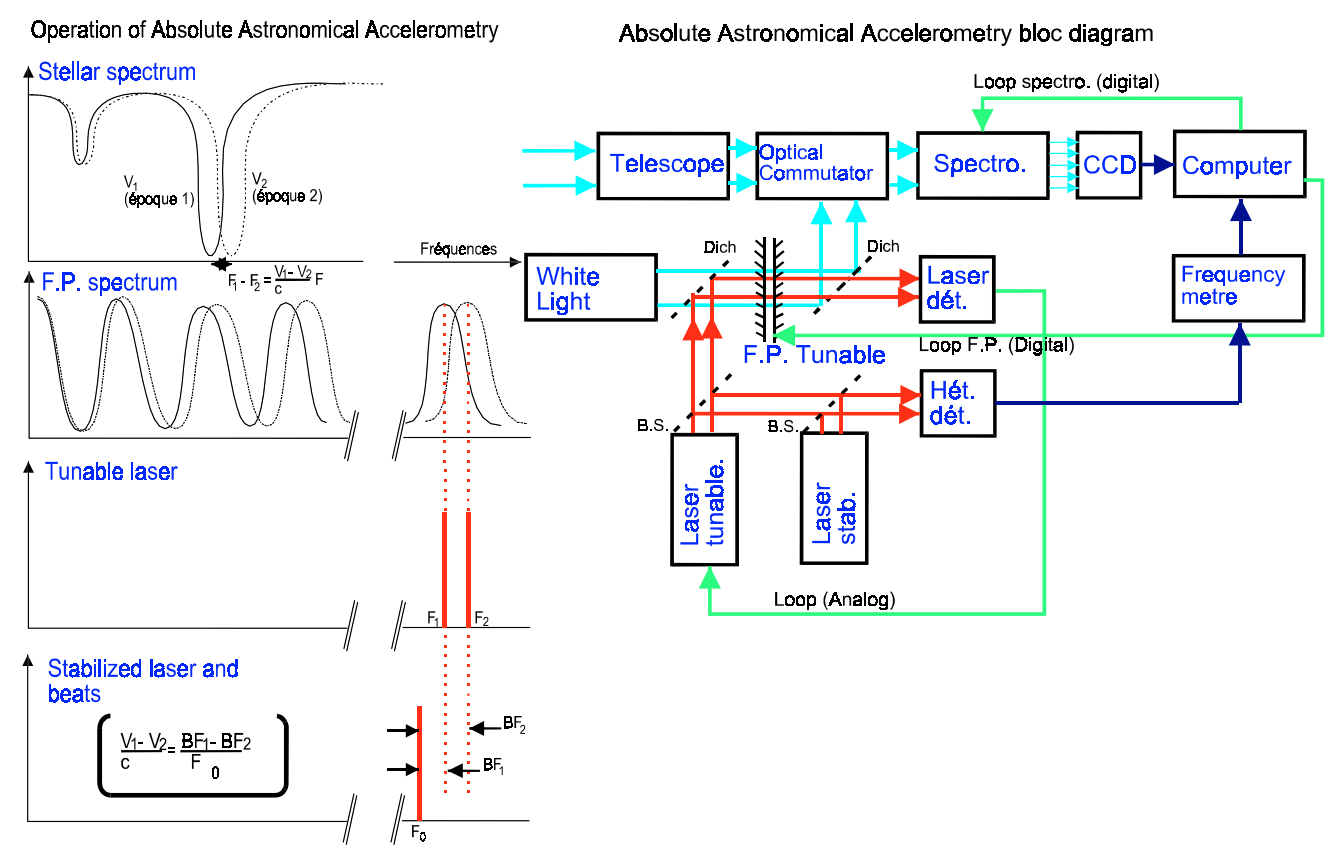

Fig. 3. Principle of the AAA system associated with the EMILIE spectrograph. On the right, the various elements of the AAA are schematized, with servo-control loops indicated. The servo-loop which controls the laser frequency is of analog type, while the others are digitally controlled through the main computer. On the left are schematized the stellar spectrum, the channelled spectrum and the beating of the two laser diodes.

Table 1. Comparison of ELODIE and EMILIE parameters for $\zeta$ Her observations.

\begin{tabular}{lll}
\hline \hline Parameter & ELODIE & EMILIE \\
\hline Spectral extent of one order $(\mathrm{nm})$ & 5.5 & 2.3 \\
Number of spectral pixels per order & 1024 & 1024 \\
Spectral size of one spectel (nm) & $5.37 \times 10^{-3}$ & $2.3 \times 10^{-3}$ \\
$V_{\mathrm{r}}$ extent for 1 spectel $\left(\mathrm{km} \mathrm{s}^{-1}\right)$ & 3 & 1.3 \\
Number of orders used & 40 & 20 \\
Spectral extent used (nm) & 220 & 46 \\
Numb. of electrons per CCD exposure & $1.64 \times 10^{9}$ & $5.23 \times 10^{8}$ \\
Exposure time (s) & 60 & 200 \\
Sampling period (s) & 98 & 252 \\
Electron flux (per s) & $2.7 \times 10^{7}$ & $2.62 \times 10^{6}$ \\
Electron flux (per s per nm) & $1.23 \times 10^{5}$ & $5.69 \times 10^{4}$ \\
Telescope collecting area $\left(\mathrm{cm}^{2}\right)$ & $2.72 \times 10^{4}$ & $1.69 \times 10^{4}$ \\
Photometric efficiency $(\%)$ & 0.59 & 0.43 \\
Photon noise on $V_{\mathrm{r}}, \delta V_{\mathrm{r}}\left(\mathrm{m} \mathrm{s}^{-1}\right)$ & 0.92 & 1.64 \\
Diameter of the fiber optics $(\operatorname{arcsec})$ & 2.0 & 2.7 \\
\hline
\end{tabular}

Note: the value of parameters depending on the wavelength are quoted here in the region $520-550 \mathrm{~nm}$.

A comparison of some of the characteristics of the two systems are summarized in Table 1, together with some parameters used during the simultaneous observations of $\zeta$ Herculis with the two systems. The principle of the AAA method is sketched in Fig. 3.

Described earlier by Connes (1993), the AAA method is intended to make the best possible use of the stellar photons, by attempting to eliminate systematic errors usually inherent to standard spectrometers. The high resolution spectrometer records a large portion of the stellar spectrum on one CCD, thanks to a cross-disperser. This spectrometer is used as a detector of the radial velocity changes of $V_{\mathrm{r}}$, induced both by a potential planetary companion, and by the much larger Earth's velocity changes (up to $\pm 30 \mathrm{~km} \mathrm{~s}^{-1}$ ). A mechanical system carrying the CCD camera inside the spectrometer is moved until the spectrum is recorded on the same pixels of the CCD detector at all epochs. Therefore the spectrometer is servo-controlled on the radial velocity of the star $V_{\mathrm{r}}$. Clearly, a very small displacement (typically $1 / 1000$ of a pixel $=1.4 \mathrm{~m} \mathrm{~s}^{-1}$ ) may be measured much more accurately than if the stellar spectrum was left to drift several tens of CCD pixels of uncontrolled widths and spacings. Similarly, a reference channeled spectrum, obtained with white light through a tunable Fabry-Pérot is recorded through the same optical path as the stellar spectrum, and in alternance with the stellar spectrum. The finesse of the FP must be small, to cover the whole CCD with a signal oscillating with wavelength, (with a typically 4 to 6 pixels periodicity). The thickness of the FP is adjusted with piezo-electric spacers in such a way that the channeled spectrum will also always remain on the same pixels as during the reference epoch. Changing the thickness of the FP results in a stretching of the white light channeled spectrum, similar to the wavelength changing of the stellar spectrum when the radial velovity $V_{\mathrm{r}}$ is changing (indeed, a Doppler stretch, $\Delta \lambda=\lambda \Delta V_{\mathrm{r}} / \mathrm{c}$, rather than the improper wording Doppler shift). The changes of thickness of the tunable Fabry-Pérot are measured with a tunable laser diode, which frequency $F$ is servo-controlled on the FP thickness. This frequency $F$ is in turn measured by heterodyne mixing with another tunable laser diode, which frequency $F_{0}$ is stabilized on an atomic cesium line, providing both the short term and long term absolute reference. The beating frequency 


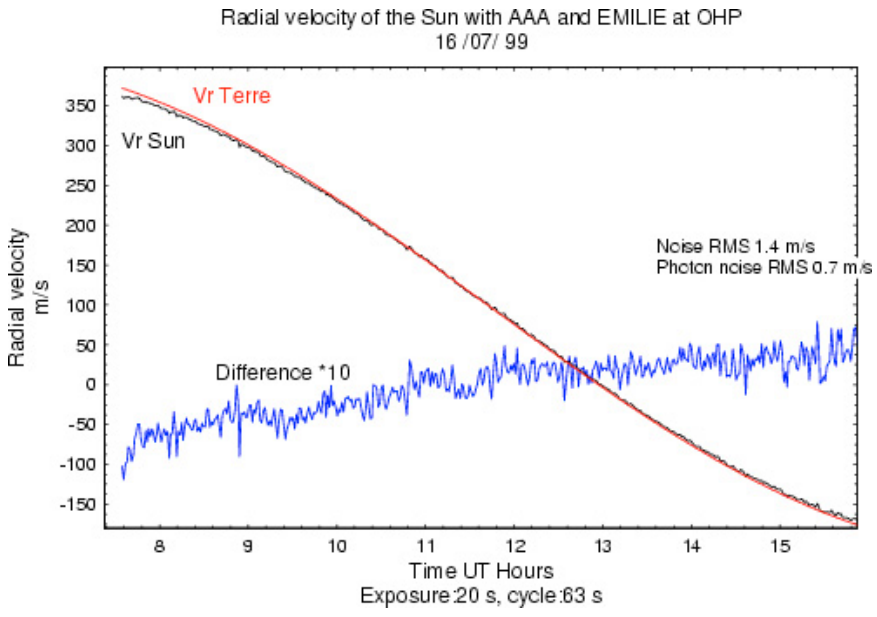

Fig. 4. Variations of radial velocity of the sun measured during one day. The continuous curve is the celestial mechanics model prediction. The variable curve shows the measurements, following the model. The difference is plotted after amplification by a factor of 10 . Solar oscillations are clearly visible in the time series, with a period around $5 \mathrm{~min}$.

$B F=F-F_{\mathrm{o}}$ is measured directly with an electronic frequency meter. The change of $V_{\mathrm{r}}$ between two epochs $\mathrm{t} 1$ and $\mathrm{t} 2$ when $V_{\mathrm{r}}=V 1$ and $V_{\mathrm{r}}=V 2$ is $V 1-V 2=c(B F 1-B F 2) / F_{\mathrm{o}}$. The barycentric velocity of the Earth must then be subtracted to get the barycentric variations of $V_{\mathrm{r}}$.

The chain of servo-control loops may be summarized below:

- The spectrometer is servo-controlled on the radial velocity of the star $V_{\mathrm{r}}$ by moving the CCD camera;

- the thickness of the FP is servo-controlled on the spectrometer, thus on $V_{\mathrm{r}}$;

- the frequency $F$ of the tunable laser is servo-controlled on the thickness of the FP, thus on $V_{\mathrm{r}}$;

- the frequency $F$ is measured with heterodyne mixing with the stabilized laser of frequency $F_{\mathrm{o}}$, and therefore the beating frequency $B F=F-F_{\mathrm{o}}$ is servo-controlled on $V_{\mathrm{r}}$.

No attempt is made to measure accurately the absolute value of the thickness of the Fabry-Pérot (typically $6 \mathrm{~mm}$ ), which would be necessary to provide an absolute wavelength reference equivalent to a set of atomic lines, allowing to measure the absolute value of $V_{\mathrm{r}}$. Rather, only changes of $V_{\mathrm{r}}$ with time are accurately tracked and measured, giving the name of accelerometry to the method.

The numerical algorithm which computes the small spectrum shift (used both for the FP and the star) makes use of the slopes of spectral features and associated variations of intensity on CCD pixels. It is much faster than a correlation algorithm, but can cope only with small displacements (a fraction of a pixel), which is enough since the spectrometer is used as a null detector.

\section{3. $V_{r}$ time-series}

The $V_{\mathrm{r}}$ are obtained after correction of the spectrometer drift, and subtraction of variations due to Earth motions (rotation and

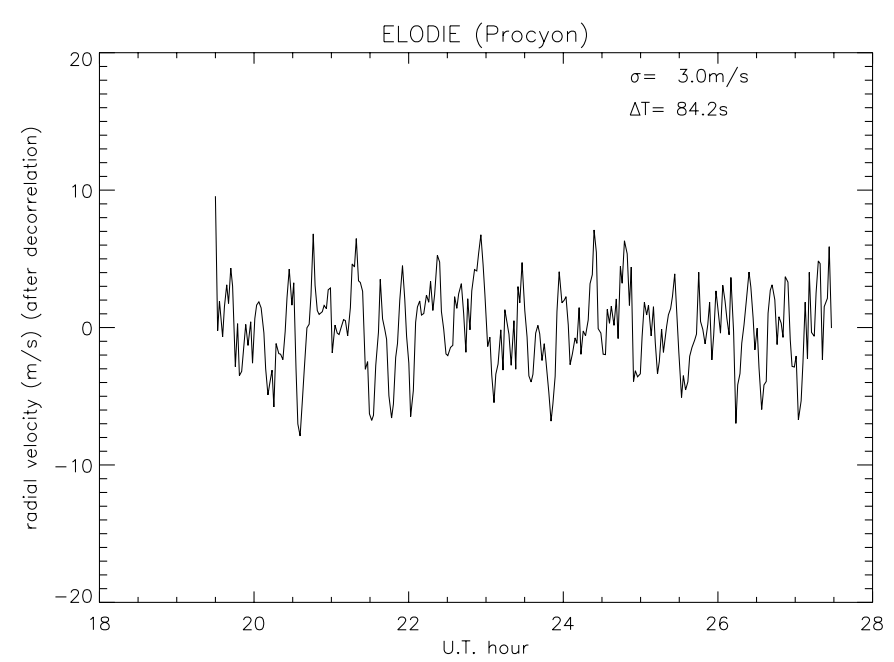

Fig. 5. Time series of Procyon obtained in January 1999 with ELODIE spectrometer.

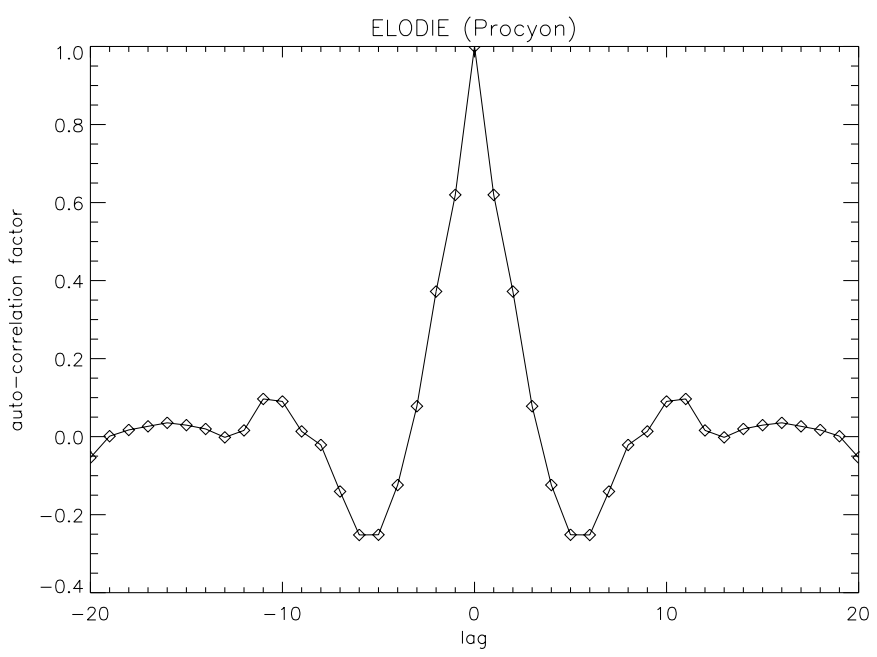

Fig. 6. Autocorrelation of the radial velocities of Procyon observed in 1999 with ELODIE.

orbital). Early test runs made on the Sun (Fig. 4) showed us that the time series of $V_{\mathrm{r}}$ were readily showing the $5 \mathrm{~min}$ oscillations "to the naked eye" that is, the signal was seen to vary with a period of about $5 \mathrm{~min}$, and an amplitude of a few $\mathrm{m} \mathrm{s}^{-1}$, much larger than the noise, and larger than any single $p$-mode amplitude (of the order of $0.2 \mathrm{~m} \mathrm{~s}^{-1}$ ). This apparently striking result stems likely from constructive interference between a small number of $p$-modes, as indicated by simulations (Barban et al. 1999). Indeed, time-series of $V_{\mathrm{r}}$ of Procyon (obtained with ELODIE) also displayed the same behavior: oscillations visible to the naked-eye, as shown by Fig. 5. A simple mathematical test may be performed on the time series of $V_{\mathrm{r}}$, in order to prove that, indeed, a given measurement is correlated to the previous measurement, or to the following measurement, as would be the case for a periodic signal sampled several times per period. If the time series were pure noise, then the correlation between one point and the following would be zero (once time trends are removed). 


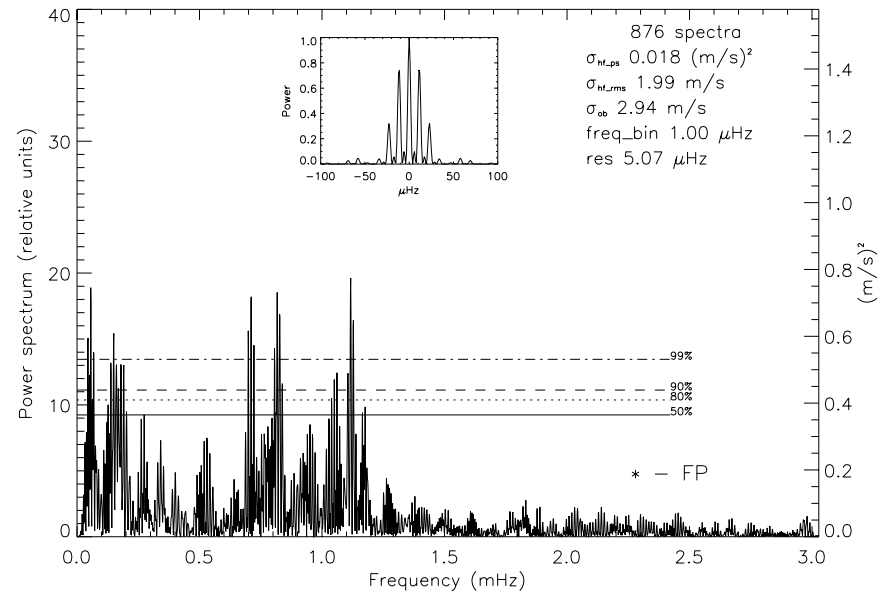

Fig. 7. Power spectrum of the Doppler shifts for Procyon (Nov. 14-16, 1998) from Martic et al. (1999).

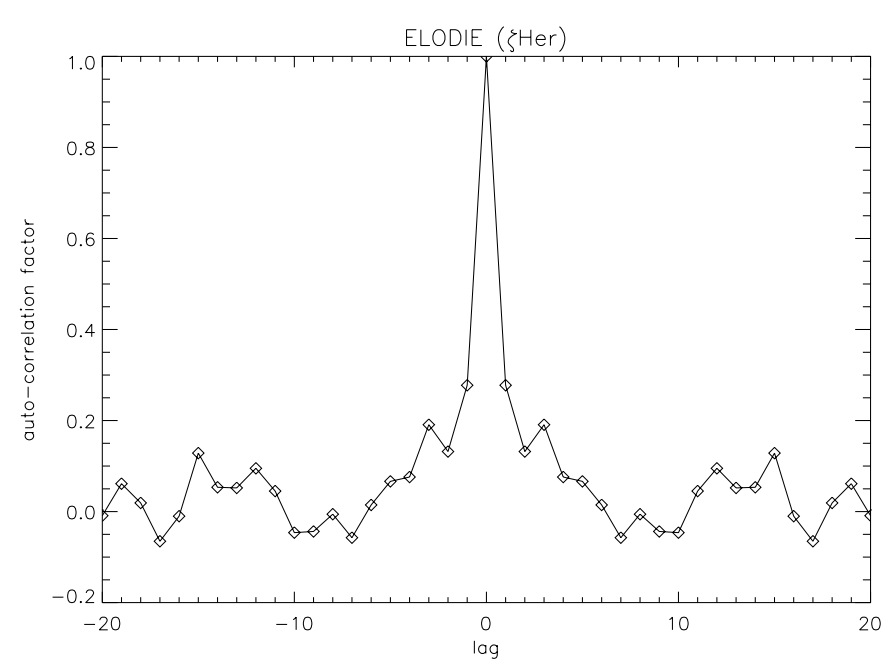

Fig. 8. Autocorrelation of the radial velocities of $\zeta$ Her observed in May 2000 with ELODIE.

We present in Fig. 6 the autocorrelation of the $V_{\mathrm{r}}$ timeseries displayed in Fig. 5, that is to say the coefficient of correlation of the value $V_{\mathrm{r}}$ of one point with another point, as a function of the lag distance between the two points (measured in number of sampled points). Obviously, for lag $=0$, the value of the auto-correlation is 1 . For lag $=1$, the coefficient is 0.62 , a very high value indeed which is genuine to the stellar signal. The correlation becomes negative with lag $=3$, and goes down (in the negative values) to -0.25 for lag $=5$ and 6 . Then it goes up again and culminates around lag $=10$. This is exactly the behavior which is expected for a periodic signal, of sinusoïdal shape, with a period of $\sim 10$ sampling intervals: $10 \times 98=980 \mathrm{~s}$, or $16.3 \mathrm{~min}$, or $\sim 1 \mathrm{mHz}$. This frequency corresponds to the average peak power found in the periodogram of Procyon derived from three consecutive nights as reproduced in Fig. 7 (Martic et al. 1999). The periodogram gives, for each frequency, the amplitude of the best fit of the time series by a single sine function.

The same exercise was done for one single night of observations for $\zeta$ Her (in Fig. 8). At lag $=1$ there is a significant

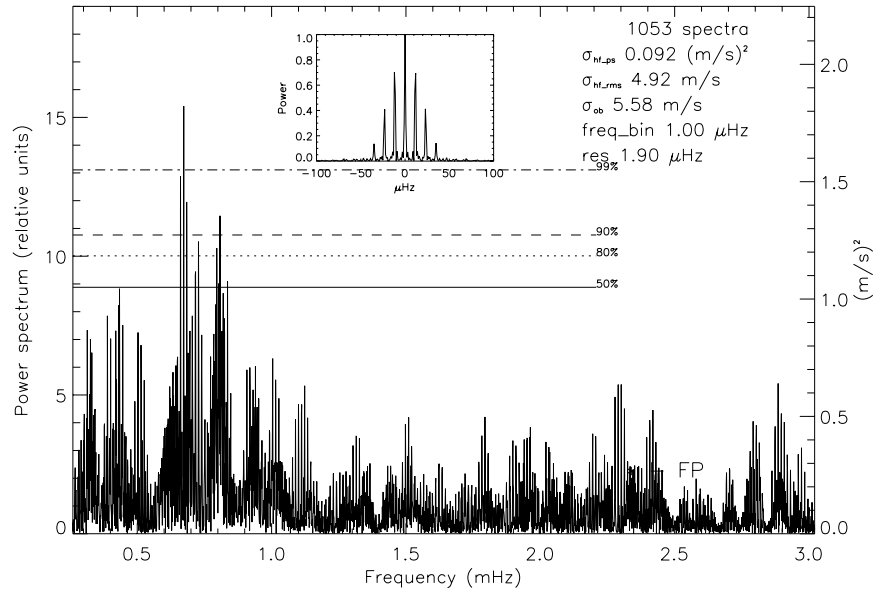

Fig. 9. Power spectrum of the radial velocity measurements of $\zeta$ Her observed in May 2000 with ELODIE (from Martic et al. 2001).

correlation of 0.28 , then decreasing, becoming negative, and positive again.

Using a sequence of Doppler shift measurements constructed from seven consecutive nights (see the resulting periodogram in Fig. 9), Martic et al. (2001) found, for $\zeta$ Her, an excess power around maximum peak at $0.7 \mathrm{mHz}$, which corresponds roughly to the period indicated by the first maximum of the autocorrelation function of Fig. 8. A refined analysis will be published separately (Martic et al. 2003).

In the upper part of Fig. 10, we present the autocorrelation of one night of $V_{\mathrm{r}}$ measurements of the star $\eta$ Cas (HR 219, $\mathrm{G} 0 \mathrm{~V}$ ). The first point at lag $=1$ gives 0.05 , that is to say no correlation (all successive points gives random numbers). Indeed, Fig. 10 (lower part) shows no obvious peak in the periodogram.

From these three examples, we can draw some preliminary conclusions:

1. The case of $\eta$ Cas with no obvious autocorrelation, indicates that indeed the autocorrelation found for the two other stars is not of instrumental origin.

2. In such a case, a large fraction of the observed fluctuations in the $V_{\mathrm{r}}$ time series are of stellar origin. This fact will be used later on to estimate the instrumental noise of the two set ups, ELODIE and EMILIE.

\section{Observations of $\zeta$ Herculis with EMILIE}

In Fig. 11, we display the raw time series of the radial velocity $V_{\mathrm{r}}$ recorded with EMILIE + AAA on $\zeta$ Herculis, during the night of 14 to 15 May 2000, together with the intensity time series. The final $V_{\mathrm{r}}$ time series, shown in Fig. 12 lower panel, has been obtained by a correction of the raw time series, by subtracting first two linear best fits for two different parts of the night, before and after $22.30 \mathrm{UT}$. Indeed, the variation of the intensity behaves differently before and after this time of the night, in a way that can be also approximated by two linear fits. Variations of the intensity $I$ are due to variations of atmospheric transparency and variations of seeing: the optical fiber accepts only $2.7^{\prime \prime}$ on the sky. Increased turbulence results in a larger stellar image, and less light entering the fiber. 


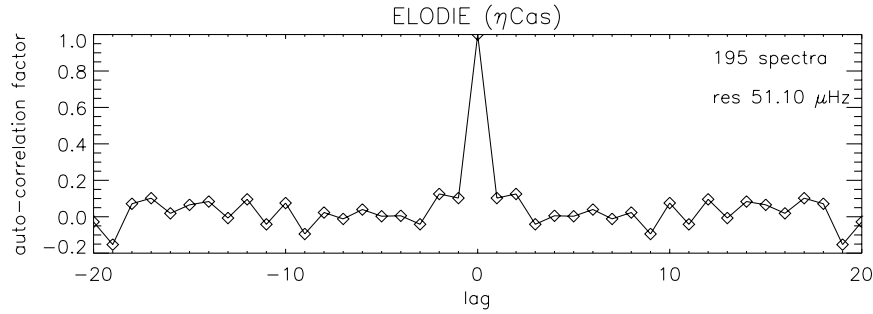

ELODIE ( $\eta$ Cas)

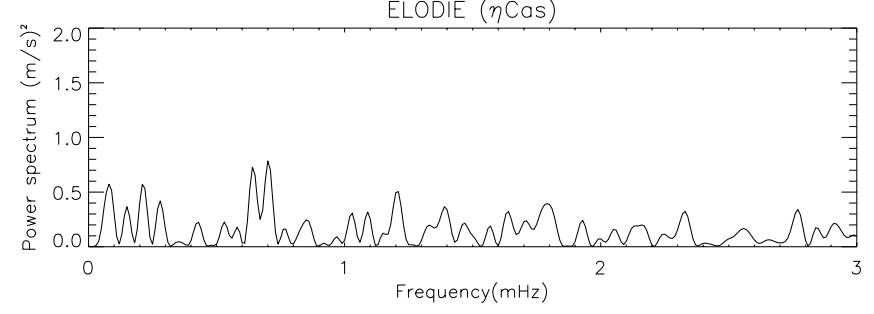

Fig. 10. Upper panel: Autocorrelation of the radial velocities of $\eta$ Cas obtained with ELODIE for one night in 1999. Lower panel: Periodogram of the same time serie.
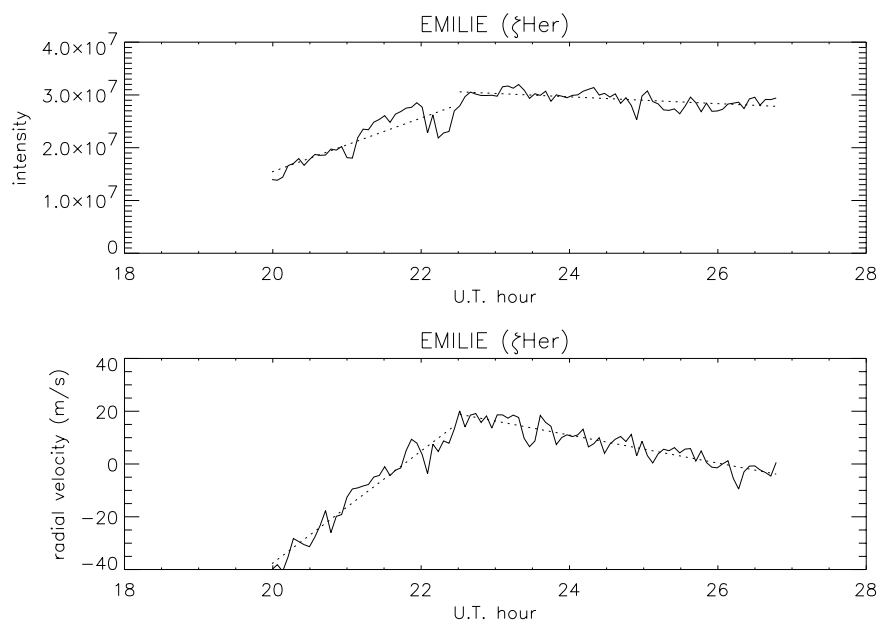

Fig. 11. Intensity and raw radial velocity of $\zeta$ Her.

The medium-term (hours) drifts observed on the uncorrected $V_{\mathrm{r}}$ time series is certainly of instrumental origin: it is correlated with intensity variations and one likely explanation is a CCD effect, in which the cross-talk of one pixel over adjacent pixels is not symmetrical, and its amount depends on the overall level of the pixel (we call this effect Asymmetric Differential Cross-Talk, ADCT). For the search for extra-solar planets, this effect is quantified, the correlation with intensity established, and a correction is applied according to the intensity to the $V_{\mathrm{r}}$ measurements. Later in the year 2000, this problem was alleviated with an automatic exposure length time system, giving a constant number of electrons in the CCD.

Here, we have adopted a simpler solution, in which we have first subtracted from the $V_{\mathrm{r}}$ (and the $I$ ) the linear fits, then established a correlation of the residual intensities and $V_{\mathrm{r}}$, and then applied a correction to the residual $V_{\mathrm{r}}$ series from the residual $I$ series. Since we are interested in the short term fluctuations, this procedure is legitimate. The resulting time series is displayed in the bottom panel of Fig. 12, with 95 points separated by $\Delta \tau=259.7 \mathrm{~s}$. The rms amplitude is $3.1 \mathrm{~m} \mathrm{~s}^{-1}$, and
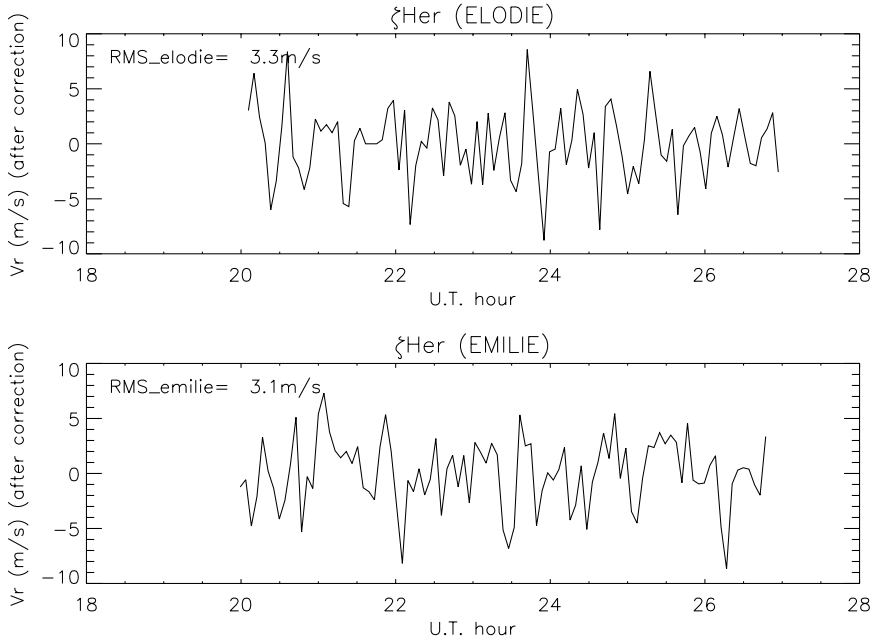

Fig. 12. Time series after decorrelation and binning for EMILIE of $\zeta$ Her.

one is tempted to see some oscillations with "the naked eye". Indeed, the auto-correlation displayed in Fig. 13 shows a value of 0.28 for lag $=1$, dropping to negative values for lag $=3$, and oscillating positive and negative, as would be expected for a sine curve. All positive peaks must correspond to increasing integer values of the period. The third positive peak is at lag $=17$, which corresponds to a frequency of $\frac{3}{17 \times 259.7}=0.68 \mathrm{mHz}$. This is in perfect agreement, both with the peak observed in the power spectrum of the EMILIE time series of Fig. 12 displayed in Fig. 14, and also with the frequency of the peak power found with ELODIE observations on the same star.

As in the case of ELODIE measurements, a significant factor for the auto-correlation at lag $=1$ is found when there is a peak in the power spectrum. The amplitude of the periodic signal is significant w.r.t. the observed fluctuations, which $\mathrm{rms}=3.1 \mathrm{~m} \mathrm{~s}^{-1}$ is in part due to genuine stellar oscillations. It could be noted that, if the raw time series (Fig. 11) had not been corrected for the linear drift, then the auto-correlation would have been always positive as was tried also; still there are a succession of larger and smaller values of the auto-correlation factor, corresponding respectively to an integer number of periods, and an odd number of half-periods. Therefore, the correction of the drifts are not changing anything fundamentally.

\section{Correlation of ELODIE and EMILIE time series}

Since the two time series of ELODIE and EMILIE, recorded simultaneously show the presence of a coherent signal at a significant level, the two time series should be correlated: they are measuring the same $V_{\mathrm{r}}$ of the $\operatorname{star} \zeta$ Her.

Since the ELODIE sampling is about three times faster than the EMILIE sampling, the ELODIE $V_{\mathrm{r}}$ measurements have been binned by three points averaged together, and both time series are compared in Fig. 12. They both present oscillations which seem to be consistent to the naked eye. The rms are 3.3 and $3.1 \mathrm{~m} \mathrm{~s}^{-1}$ respectively for ELODIE and EMILIE. The auto-correlation of each curve are represented in Fig. 13, and are very similar. 


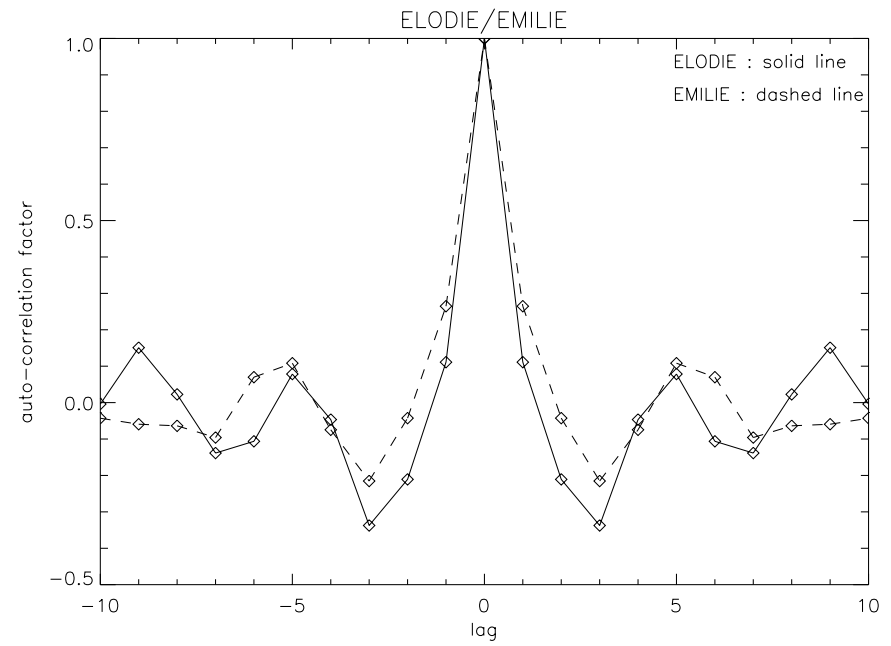

Fig. 13. Autocorrelation of the radial velocities of $\zeta$ Her obtained with ELODIE and EMILIE.
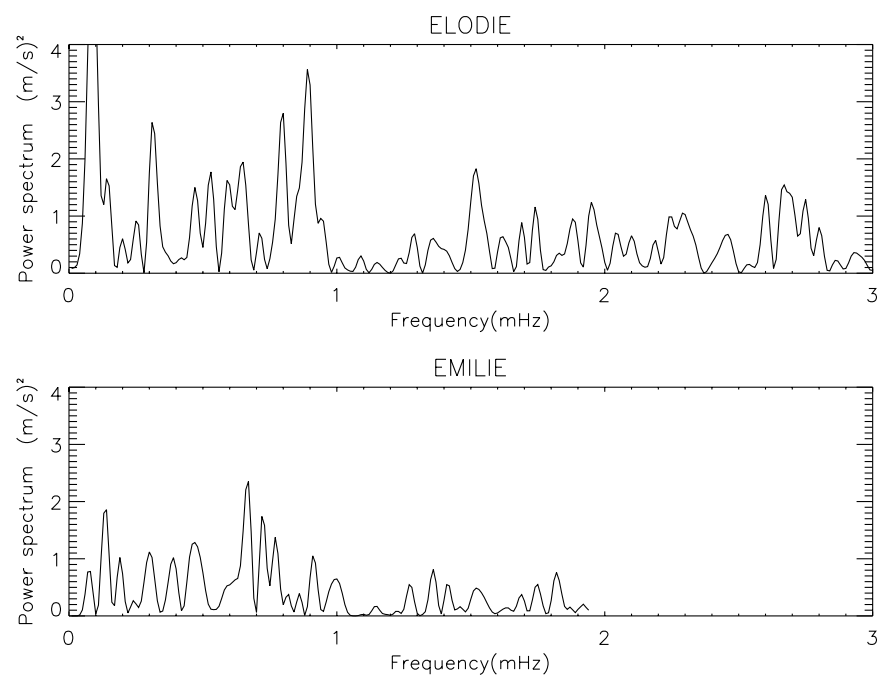

Fig. 14. Power spectrum of the radial velocities of $\zeta$ Her obtained with ELODIE and EMILIE.

In Fig. 15, we plot the $V_{\mathrm{r}}$ value recorded by EMILIE, as a function of the value recorded with ELODIE at the same time (by linear interpolation into the binned ELODIE series). The cloud of points is widely spread, but the linear fit shows a slope of 0.203 , with a $1 \sigma$ error on this slope of 0.128 . Therefore, the slope is definitely real.

In Fig. 16 upper panel, the cross-correlation between the two series is shown as a function of the lag between the respective points of the two series, sampled at the times of EMILIE series. Both for lag $=0$ and lag $=-1$, the correlation is positive, while it becomes negative for lag $=-3$ and +2 . Quite clearly, this dissymetry w.r.t. lag $=0$ suggests a datation error in the relative timing of both series. Indeed, this could be tracked back to the fact that the timing of EMILIE series was taken as the starting time of the exposure, instead of the mid time of the exposure for ELODIE. The lower panel of Fig. 16 indicates the same cross-correlation with an oversampling factor of 4 , for a better determination of the datation error.

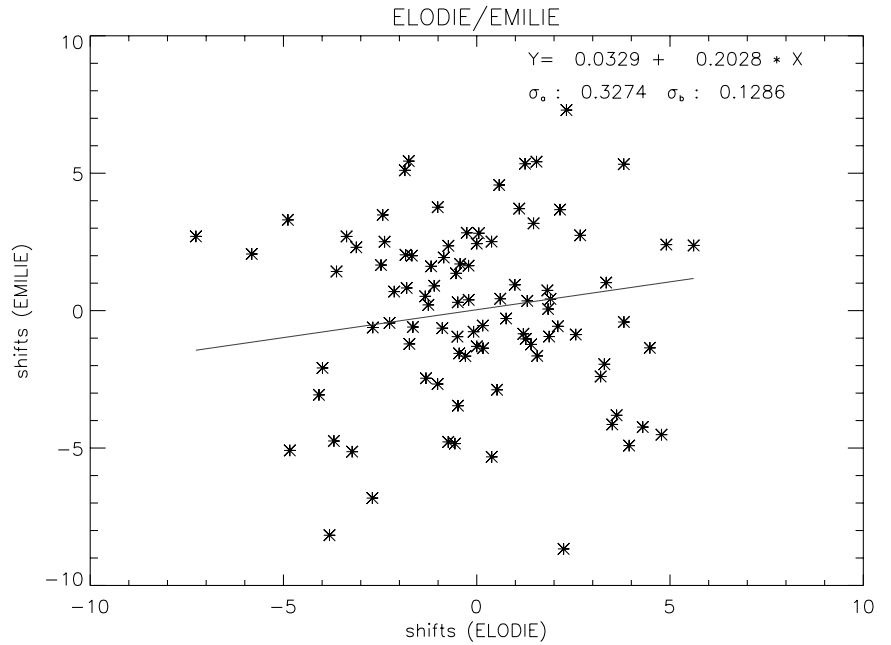

Fig. 15. Correlation ELODIE/EMILIE from $V_{\mathrm{r}}$ time series of $\zeta$ Her.
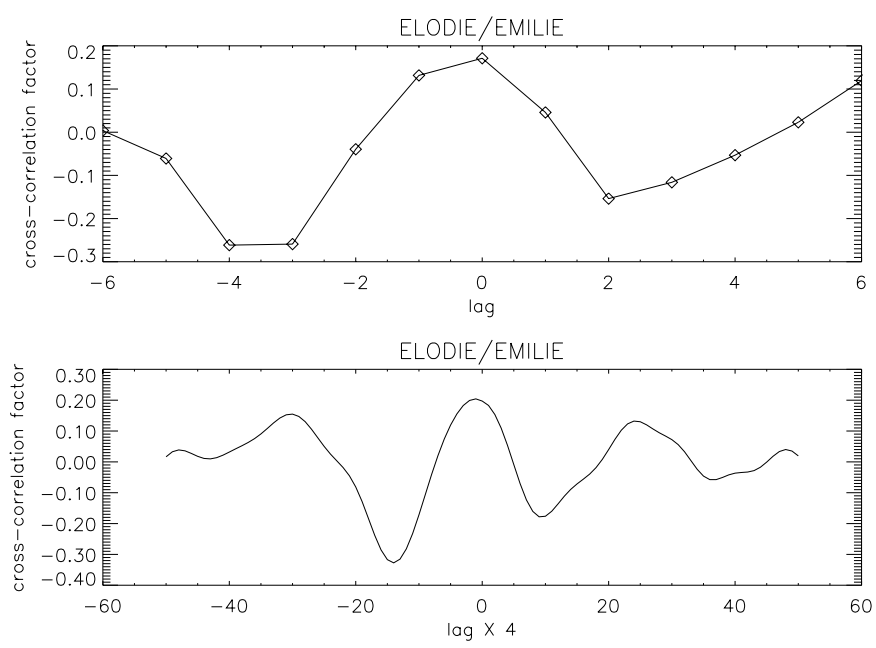

Fig. 16. Cross-correlation between the two time series of $\zeta$ Her observed in May 2000 with ELODIE and EMILIE. The lower panel is made with an oversampling factor of 4 .

This analysis shows that the two instruments have independently recorded a coherent signal, with the same phase in the two time series, which is of stellar origin. We believe that it is the first time that such stellar oscillations on a solar type star are recorded simultaneously, with two different set ups, and display a consistent behaviour in the two time series.

Though the two instruments are at the same geographic location, the same type of analysis could apply to simultaneous observations from two sites well separated in longitude. If there is some overlap of the two time series, the correlation analysis between them should be performed for two reasons:

- to detect small relative datation errors in the two time series;

- to check that there is indeed a central peak at lag $=0$.

If there are no peak, it means that the periodic signal is significantly smaller than the noise. And, as it was seen in previous sections, there is less hope of finding a significant peak in the Fourier analysis under such adverse circumstances. 
Table 2. Variances and $\sigma_{\text {rms }}$ of radial velocities.

\begin{tabular}{lllll}
\hline \hline & $m_{\mathrm{V}}$ & $\begin{array}{l}V_{\text {ar(obs) }} \\
\left(\mathrm{m} \mathrm{s}^{-1}\right)^{2}\end{array}$ & $\begin{array}{l}\sigma_{\mathrm{obs}} \\
\mathrm{m} \mathrm{s}^{-1}\end{array}$ & $\begin{array}{l}V_{\text {ar(phot) }} \\
\left(\mathrm{m} \mathrm{s}^{-1}\right)^{2}\end{array}$ \\
\hline$\alpha$ CMi(ELODIE) & 0.34 & 10 & 3.1 & - \\
$\zeta$ Her(ELODIE) & 2.81 & 31 & 5.6 & 0.92 \\
After binning & 2.81 & 10.9 & 3.3 & 0.28 \\
$\eta$ Cas(ELODIE) & 3.45 & 6 & 2.45 & 1 \\
$\zeta$ Her(EMILIE) & 2.81 & 10 & 3.1 & 2.69 \\
\hline
\end{tabular}

\section{5. $V_{r}$ noise analysis for the detection of stellar oscillations}

In this section, we try to quantify the various sources resulting in the observed fluctuations of $V_{\mathrm{r}}$ time-series recorded along a single night, relevant to the detection of stellar oscillations. Since a typical sampling period for asteroseismology is of the order of $1 \mathrm{~min}$, here we ignore longer term fluctuations: hours and days, which could come either from the instrument, or from the star itself. A dark spot (like a solar spot) appearing on one side of the star is decreasing the light coming from the proceeding side of the star, and will influence the disk integrated radial velocity. But this influence will evolve at the rotation rate of the star, that is to say on days scale time. This kind of problem, producing a slow drift of $V_{\mathrm{r}}$, detrimental in the case of extra-solar planet search is easily coped with for the detection of stellar oscillations through Fourier transform analysis. The same is true for instrumental drifts on hours/days time-scale.

The (short term) observed fluctuations of $V_{\mathrm{r}}$ result from three different sources:

- the genuine fluctuations of the star (including the oscillations);

- the photon noise of each single measurement;

- all other sources of instrumental noise, including the possible atmospheric effects (turbulence, seeing, variable atmospheric transmission, differential refraction...).

Since the three sources are independent, we may write, using the variance $V_{\mathrm{ar}}=\sigma^{2}$ :

$V_{\mathrm{ar}(\mathrm{obs})}=V_{\mathrm{ar}(\mathrm{phot})}+V_{\mathrm{ar}(\mathrm{star})}+V_{\mathrm{ar}(\mathrm{inst}+\mathrm{atm})}$

with obvious notations. This equation is valid, not only for the time-series of individual measurement, but also if these individual measurements are binned by 2,3 , or more.

The photon noise $\delta V_{\mathrm{r}}=\sqrt{V_{\text {ar(phot) }}}$, that affects a single measurement is estimated by the following expression (Connes 1985).

$\delta V_{\mathrm{r}}=\frac{c}{Q} \frac{1}{\sqrt{N_{\mathrm{e}}}}$

where $c$ is the velocity of light, $N_{\mathrm{e}}$ the total number of electrons recorded on the CCD and used in the calculation of $\delta V_{\mathrm{r}}$, and $Q$ a quality factor quantifying in a precise way (see Connes 1985) the power of the various absorption lines in the stellar spectrum in order to be able to measure a Doppler shift of this spectrum.

For the three solar-type stars considered in this study, $Q$ is of the order of 8000 , and $\delta V_{\mathrm{r}}$ is indicated in Table 2, as well

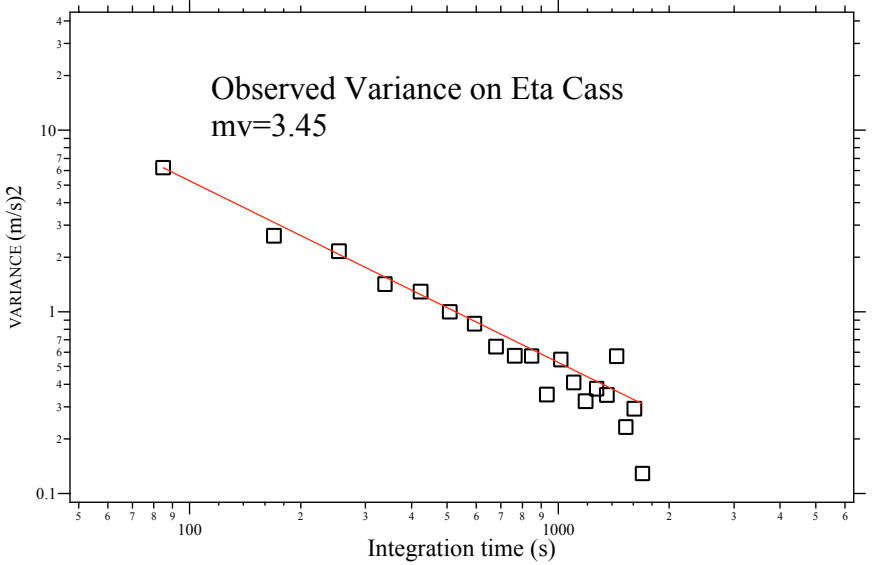

Fig. 17. Observed variance on $\eta$ Cas.

as $V_{\text {ar(obs) }}$ for the three stars considered. For the 3 stars observed with ELODIE, the lowest observed fluctuations are for $\eta$ Cas, the dimmest star. This means that a large part of the observed variance for Procyon and $\zeta$ Her is of genuine stellar origin: the stellar oscillations, as demonstrated also by the periodogram analysis.

If we assume that the star $\eta$ Cas is very quiet, with no oscillations or intrinsic fluctuations, then we may apply formula (1) with $V_{\text {ar(star) }}=0$, yielding an upper limit for the instrument + atmosphere noise:

$V_{\text {ar(inst + atm })}<5\left(\mathrm{~m} \mathrm{~s}^{-1}\right)^{2} \quad \sigma_{(\text {inst }+ \text { atm })}<2.23 \mathrm{~m} \mathrm{~s}^{-1}$

for an integration time of $60 \mathrm{~s}$. The $\eta$ Cas time series recorded on one night in 1998 with ELODIE has been binned and averaged by $2,3, \ldots$ up to 20 points, and the rms calculated again. This rms is plotted as a function of the integration time (resulting from the bin size) in Fig. 17, together with a straight line of slope -1 starting for the rms observed with single measurements. The points are following the straight line, showing that the errors connected to the instrument + atmosphere have a Gaussian behavior, at least up to $\sim 0.5 \mathrm{~h}$ integration time where the variance goes down to $0.3\left(\mathrm{~m} \mathrm{~s}^{-1}\right)^{2}$. If we extrapolate to $\sim 10^{5} \mathrm{~s}$, (a reasonable duration for asteroseismology measurements) then this source of noise will go down to $2.23 / \sqrt{10^{5} / 60}=0.05 \mathrm{~m} \mathrm{~s}^{-1}$. This is a factor of two below the amplitude of solar oscillations, which implies a great prospect in the possibility of high-accuracy $V_{\mathrm{r}}$ measurements with the Fabry-Pérot technique in asteroseismology, especially if a longitude-spread network can be implemented.

The ELODIE observations of $\eta$ Cas $\left(m_{\mathrm{v}}=3.45\right)$ yield a photon variance of $1 \mathrm{~m} \mathrm{~s}^{-1}$, a factor of 5 smaller than the variance due to instrument + atmosphere noise. Therefore, the actual limitation for asteroseismology on ELODIE is the instrument+atmosphere noise, for a star of $m_{\mathrm{v}}=3.45$. This noise amplitude is most likely independent of the star's magnitude. Dimmer stars could be observed with ELODIE with about the same performance. With the present efficiency of ELODIE spectrometer $(\sim 0.6 \%$ average) the photon noise will reach the instrument + atmosphere noise $\left(V_{\mathrm{ar}}=5\left(\mathrm{~m} \mathrm{~s}^{-1}\right)^{2}\right)$ with a star magnitude $3.45+2.5 \log 5=5.2$ for a $60 \mathrm{~s}$ exposure time. New spectrometers are claiming efficiencies of about $10 \%$ (FEROS 
at ESO), possibly at the expense of short term stability considerations; however, it is quite clear that a new spectrometer could have the same short term stability as ELODIE, and have an efficiency $\sim 5$ times better (reaching 3\%) than the present efficiency of ELODIE. With the help of the fixed Fabry-Perot technique demonstrated with ELODIE, the photon noise would reach the atmosphere+instrument noise $\left(V_{\mathrm{ar}}=5\left(\mathrm{~m} \mathrm{~s}^{-1}\right)^{2}\right)$ for a $60 \mathrm{~s}$. exposure time, and star magnitudes of $\sim 7$. This would open a new and interesting field to asteroseismology, to be performed from the ground, especially if a reasonable network can be implemented on medium size telescopes. However, it requires absolutely the high spectroscopic stability provided by the fixed Fabry-Pérot technique demonstrated with ELODIE or EMILIE.

\section{Comparison EMILIE/ELODIE}

For the night of simultaneous recording of $V_{\mathrm{r}}$ time series on $\zeta$ Herculis, we may apply formula (1) to each instrument, and take into account the observed variance and estimated photon noise of Table 2:

For ELODIE:

$$
10.9=0.28+V_{\mathrm{ar}(\mathrm{star})}+V_{\mathrm{ar}(\mathrm{inst}+\mathrm{atm}) \text { elodie }}
$$

\section{For EMILIE:}

$$
9.61=2.69+V_{\text {ar(star })}+V_{\text {ar(inst }+ \text { atm }) \text { emilie }} .
$$

Since $V_{\mathrm{ar}(\mathrm{star})}$ is the same for the two series of observations, it comes:

$V_{\text {ar(inst }+ \text { atm)elodie }}=V_{\text {ar(inst }+ \text { atm }) \text { emilie }}+3.7$.

It shows that the EMILIE instrument is significantly better than ELODIE, as far as the short term instrumental noise is concerned. We can go one step further, by estimating $V_{\text {ar(star). A }}$ lower estimate of $V_{\text {ar(star) }}$ is given by the squared amplitude of the highest peak in the periodogram of Fig. 9, at $1.8\left(\mathrm{~m} \mathrm{~s}^{-1}\right)^{2}$. It assumes that the star signal would be a pure sinewave of $1.8\left(\mathrm{~m} \mathrm{~s}^{-1}\right)^{2}$ squared amplitude. Then, we derive an upper estimate of the instrument atmosphere + variances:

$$
\begin{array}{ll}
V_{\text {ar(inst + atm)emilie }}<5.12 & \sigma_{\text {emilie }}<2.26 \mathrm{~m} \mathrm{~s}^{-1} \\
V_{\text {ar(inst }+ \text { atm)elodie }}<8.82 & \sigma_{\text {elodie }}<2.97 \mathrm{~m} \mathrm{~s}^{-1} .
\end{array}
$$

This is a significant difference, considering also the fact that only a lower estimate of $V_{\mathrm{ar}(\mathrm{star})}$ was taken. A larger $V_{\mathrm{ar}(\mathrm{star})}$ would amplify the difference in $\sigma$.

We may also compare $V_{\text {ar(inst + atm) }}$ for the two ELODIE observations of $\eta \mathrm{Cas},<5\left(\mathrm{~m} \mathrm{~s}^{-1}\right)^{2}$, and $\zeta$ Her around $28\left(\mathrm{~m} \mathrm{~s}^{-1}\right)^{2}$, derived from the estimate of $V_{\text {ar(star })}=1.8\left(\mathrm{~m} \mathrm{~s}^{-1}\right)^{2}$ for $\zeta \mathrm{Her}$ and application of formula (1) for $\zeta$ Her ELODIE with no time binning.

This points clearly to a night-to-night variation of the (instrument+atmosphere) noise, and we believe that this is due to atmospheric conditions. Indeed, it was observed with ELODIE that the (instrument+atmosphere) noise is smaller when the intensity variations are smaller, probably as a result of a more constant seeing. Variations of seeing, combined with atmospheric differential refraction, and imperfect guiding and scrambling, are possibly the cause of the residual (instrumental+atmosphere) noise.

Similar day to day variations of the observed rms (after correction of drifts) were reported (Carrier et al. 2001) for the CORALIE spectrometer on the $1.2 \mathrm{~m}$ Swiss telescope at La Silla: from 2.0 to $3.3 \mathrm{~m} \mathrm{~s}^{-1}$, for $\beta$ Hyi $\left(m_{\mathrm{v}}=3.0\right)$, comparable to our $3.1 \mathrm{~m} \mathrm{~s}^{-1} \mathrm{rms}$ observed with EMILIE on $\zeta$ Her (same visual magnitude).

\section{Conclusions}

We have developed and implemented a new technique allowing one to perform asteroseismologic measurements from the ground in an efficient manner. Based on the ideas of Connes (1984, 1985), it makes use of a white light channeled spectrum through a Fabry-Pérot as a calibration system to achieve high accuracy measurements of radial velocity variations, through Doppler shift of stellar spectra displaying numerous absorption lines.

Two variants of this technique were implemented and tested at Observatoire de Haute Provence:

- a simple, fixed Fabry-Pérot with a tungsten lamp was placed as an external calibration system of the two-fiber ELODIE spectrometer since 1994 (at the $1.93 \mathrm{~m}$ telescope). This system led to the first unambiguous detection of stellar oscillations of the star Procyon in 1998 (Martic et al. 1999);

- the full AAA system, with a tunable Fabry-Pérot system whose thickness is controlled by tunable laser diodes, implemented at the $1.52 \mathrm{~m}$ telescope, associated with the EMILIE spectrometer.

The noise performance of the two set-ups were derived from the analysis of the fluctuations of single-night time series of 3 stars. The star $\eta$ Cas is the most quiet of the 3 stars (smallest short-term fluctuations), and may serve as standard of quietness. Observed with the fixed FP, an upper estimate of the noise introduced by the instrument and by the atmosphere was obtained at less than $2.45 \mathrm{~m} \mathrm{~s}^{-1}$ for a $60 \mathrm{~s}$ exposure (not including photon noise). However, the level of noise seems to depend on the night quality, showing $\sim 3 \mathrm{~m} \mathrm{~s}^{-1}$ in some other circumstances. Further studies of this problem could yield a smaller sensitivity of the (instrument+atmosphere) noise to atmospheric conditions, mainly through software improvements and better scrambling.

From the simultaneous observation of star $\zeta$ Herculis, it is shown that the (instrument+atmosphere) noise is smaller for EMILIE $\left(\leq 2.1 \mathrm{~m} \mathrm{~s}^{-1}\right)$ than for ELODIE $\left(\leq 2.8 \mathrm{~m} \mathrm{~s}^{-1}\right)$. Still, the ELODIE system collects more photons (about 10 times more electrons created) and this compensates for that. However, a new backside illuminated CCD was implemented in August 2001, in the EMILIE spectrometer, which might change the situation.

We believe that we detected for the first time in a solartype star a correlated signal in the time series of $V_{\mathrm{r}}$ observed simultaneously with two different instruments, a situation that 
is similar to the observation with a network of telescopes spread in longitude, suggesting a great potential for the Fabry-Pérot technique of calibration applied in a network of telescopes. We provide data suggesting that the full AAA system is better performing than the more simple fixed Fabry-Pérot technique, mostly because the AAA is servo-controlled and many sources of small errors are cancelled out. Further investigations and observations seem warranted for such a system.

Finally, we suggest a simple autocorrelation test on a time series of $V_{\mathrm{r}}$ measurements as a first exercise to evaluate its potential for the derivation of oscillations, as soon as it has been recorded.

\footnotetext{
Acknowledgements. Our gratitude goes first to Pierre Connes, for having started the whole project of measuring radial velocities of stars with a high precision, with the help of Fabry-Pérot as a calibration system, and its further development with EMILIE/AAA. Then, we thank Milena Martić for her major contribution in the ELODIE asteroseismology program, and for valuable discussions. In particular, she modified extensively the standard ELODIE software to implement an asteroseismic mode in which a long series of short exposures are recorded automatically. We are indebted to Arlan Mantz for providing the replica echelle grating for EMILIE. We thank Jean-Pierre Sivan, Director of Observatoire de Haute-Provence, for his support on the whole EMILIE/AAA project, and many members of the OHP staff who helped us. We also thank Geneviève Debouzy for her support as Deputy Director of INSU, and INSU/CNRS for financing both projects with Fabry-Pérot Technique, the french Ministry of Research and Technology(MENRT) for partial financing of EMILIE/AAA, PNPS (Programme National en Physique Stellaire) for financing the observation telescope cost, and Thierry Appourchaux from ESA for a contribution to the ELODIE Fabry-Pérot.
}

\section{References}

Ando, H., Watanabe, E., Yutani, M., Shimizu, Y., \& Nishimura, S. 1988, PASJ, 40, 249

Baranne, A., Queloz, D., Mayor, M., et al. 1996, A\&AS, 119, 373

Barban, C., Michel, E., Martic, M., et al. 1999, A\&A, 350, 617

Bedford, D. K., Chaplin, W. J., Coates, A. R., et al. 1995, MNRAS, 273, 367

Baglin, A., The Equation of State in Astrophysics 1994, ESA Conf. 512 B, IAU Colloq., 147

Bedding, T. R., Butler, R. P., Kjeldsen, H., et al. 2001, ApJ, 549, L105

Bouchy, F. 1999, Ph.D. Thesis, Université Paris VII, Paris, France

Bouchy, F., \& Connes, P. 1999, A\&AS, 136, 193

Bouchy, F., \& Carrier, F. 2001, A\&A, 374, L5

Christensen-Dalsgaard, J. 1998, Ap\&SS, 261, 1

Carrier, F., Bouchy, F., Kienzle, F., et al. 2001, A\&A, 378, 142

Connes, P. 1985, Ap\&SS, 110, 211

Connes, P. 1993, Ap\&SS, 212, 357

Connes, P., Martic, M., \& Schmitt, J. 1996, Ap\&SS, 241, 61

Gelly, B., Grec, G., \& Fossat, E. 1986, A\&A, 164, 383

Gilliland, R. L., Brown, T. M., Kjeldsen, H., et al. 1993, AJ, 106, 2441

Innis, J. L., Isaak, G. R., Speake, C. C., Brazier, R. I., \& Williams, H. K. 1991, MNRAS, 249, 643

Kjeldsen, H., Bedding, T. R., Frandsen, S., \& Dall, T. H. 1999, MNRAS, 303, 579

Martic, M., Lebrun, J. C., Schmitt, J., \& Bertaux, J. L. 2000, IAU, 203, 121

Martic, M., Lebrun, J. C., Schmitt, J., Appourchaux, T., \& Bertaux, J. L. 2001, ESA SP, 464, 431

Martic, M., Schmitt, J., Lebrun, J. C., et al. 1999, A\&A, 351, 993

Martic, M., et al. 2003, in preparation

Mayor, M., \& Queloz, D. 1995, Nature, 378, 355

Schmitt, J., 1997, Étude et réalisation en laboratoire d'un accéléromètre astronomique absolu, Thèse de Doctorat, Université Paris VI

Schmitt, J., \& Bertaux, J. L. 2003, in preparation 